\title{
A Profile Measurement System for Rail Quality Assessment during Manufacturing
}

\author{
Julio Molleda*, Rubén Usamentiaga*, Álvaro F. Millara*, Daniel F. García*, Member, IEEE, Pedro Manso*, \\ Carlos M. Suárez ${ }^{\dagger}$, Ignacio García ${ }^{\ddagger}$ \\ *Department of Computer Science and Engineering, University of Oviedo, Spain \\ ${ }^{\dagger}$ Department of Manufacturing Engineering, University of Oviedo, Spain

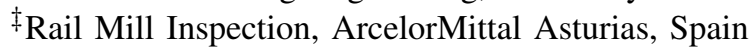

\begin{abstract}
Steel rails used in the transport sector and in industry are designed and manufactured to support high stress levels generated by high-speed and heavy-loaded modern trains. In the rail manufacturing process, one of the key stages is rolling, where fast, accurate and repeatable rail profile measurement is a major challenge. In this paper, a rail profile measurement system for rail rolling mills based on four conventional, inexpensive laser range finders is proposed. The range finders are calibrated using a common reference to properly express the point clouds generated by each range finder in the world coordinate system. The alignment of the point clouds to the rail model is performed by means of an efficient and robust registration method. Experiments carried out in a rail rolling mill demonstrate the accuracy and repeatability of the system; the maximum error is below $0.12 \%$. All parallelizable tasks were designed and developed to be executed concurrently, achieving an acquisition rate of up to 210 fps.
\end{abstract}

Keywords-Industrial inspection; profile measurement; machine vision; rage imaging; camera calibration.

\section{INTRODUCTION}

Steel rails are used in the transport sector as well as in a wide variety of industries. In these scenarios, the high speed and heavy loads of modern trains require rail tracks designed and manufactured to support high stress levels. In the manufacturing process, one of the key stages is rolling. At the end of this stage rails must be inspected to verify that they meet the dimensional quality standards required by the customer. Current high-quality standards define tight shape tolerances, which must be met while maintaining high productivity rates. Thus, modern rail mills cannot be conceived without a number of machine-vision-based systems for monitoring and controlling the rolling process. In recent decades this type of tasks has been automated [1] and a wide range of $3 \mathrm{D}$ techniques for imaging and visualization in industrial environments has been proposed [2]. The development of machine vision devices at decreased costs, their miniaturization and their integration in industrial processes have increased the use of 3D imaging systems in industry [3]. Rail rolling mills, as well as many other industries where meeting the current demand for high quality standards is essential, have benefited from the use of 3D imaging.

Usually, 3D data acquisition techniques are classified into contact and non-contact techniques. The former touch the surface of the object by means of a mechanical sensor, whereas the latter use non-contact devices based on magnetic, optical or acoustic principles. Non-contact techniques are commonly divided into passive, which use environmental or ambient light to illuminate the scene, and active, which control the illumination of the scene by projecting light patterns onto it [4]. The most commonly used 3D imaging systems in industry are based on active techniques, especially when these systems are required to work in harsh environments where passive methods are negatively affected by ambient or surrounding light.

Rails are systematically inspected for surface defects and wear using non-destructive evaluation [5]. Rail inspection is carried out in the manufacturing process in the rolling mill for quality assessment and assurance. Furthermore, rails are inspected while the railway tracks are operated in order to detect defects due to surface cracks, plastic deformations and wear. Contact-based rail inspection systems can apply tactile techniques, using mechanical devices with several leverages, to indirectly observe the rail geometry. Some other contactbased systems measure vertical and horizontal accelerations using moving vehicles [6]. In the recent years, systems tend to use non-contact techniques, mainly based on automated visual inspection using structured light [7], [8], [9], [10], [11].

In this paper, a machine-vision-based, non-contact profile measurement system (PMS) of rails for rail rolling mills is presented [12]. This system provides in-process measurements of several dimensions of rails based on geometric parameters of the transverse sections, also called profiles, of these rails. The PMS is based on active range imaging, specifically on laser triangulation, using four coupled laser range finders, similar to those used in other applications in the metal industry [13]. Experiments carried out both in the lab and in a rail mill demonstrate that the PMS provides accurate and repeatable measurements. The presented system has three main contributions: 1) Accurate rail quality inspection in rolling mills using conventional and inexpensive machine vision components; 2) A robust and accurate calibration procedure; 3) An efficient and robust method to align point clouds obtained from multiple range finders to rail models.

The reminder of this paper is organized as follows: Section II presents the the proposed approach; Section III describes system calibration and assessment; Section IV discusses the results obtained in the experiments; and finally, Section V reports conclusions.

\section{PROPOSED APPROACH}

Designing a dimensional inspection system for rail manufacturing involves many factors: measurement volume, reli- 


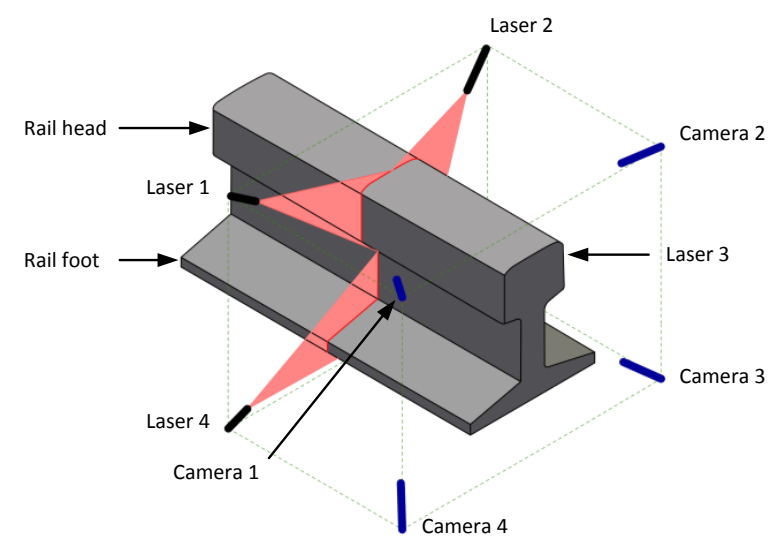

Fig. 1. Geometry of the proposed rail profile measurement system.

ability, accuracy, repeatability, cost, and computational speed, among others. Furthermore, the system may be constrained by environmental issues such as ambient light, temperature, humidity and dust. These factors and constraints are determinant in the geometry as well as the hardware and software components of the system.

\section{A. Geometry}

The rail PMS proposed in this paper uses four coupled laser range finders, as shown in Fig. 1 (laser emitter \#3 is hidden because of the perspective). The location of the laser range finders is determined taking into account the dimensions of the rail head and foot (see Fig. 1), minimizing the occluded areas of the rail surface during the inspection process.

\section{B. Active triangulation components}

Each range finder is equipped with a CCD matrix camera: a DALSA Genie HM1400, featuring 1400x1024 pixel resolution. Each camera is equipped with a high-resolution, $16 \mathrm{~mm}$, Goyo GMTHR21614MCN lens. These cameras use a GigE Vision interface, a quad-port Intel Ethernet Server Adapter I350, connected to the host computer. On the other hand, each light pattern projected onto the rail surface is a single line generated by a Lasiris emitter with a diode power of $30 \mathrm{~mW}$ and a fan angle of $30^{\circ}$. Adjacent laser emitters project the laser pattern with different wavelengths, $660 \mathrm{~nm}$ and $685 \mathrm{~nm}$. Thus, using an optical filter each camera is able to easily image the pattern projected by its counterpart emitter, mitigating the effect of the patterns generated by adjacent lasers.

\section{Measurement pipeline}

Fig. 2 depicts the five main stages of the PMS described in this paper: image acquisition, laser line detection and extraction, $2 \mathrm{D}$ to $3 \mathrm{D}$ coordinate translation, 3D rail profile computation, and dimensional measurement of the rail profile. The first three stages are replicated since they can run in parallel over independent data, following an MIMD approach. Each stage is a producer/consumer implemented following the pipeline pattern using parallel tasks and queues. Each task implements a stage of the pipeline, and the queues act as buffers that allow the stages of the pipeline to run concurrently, even though images are processed in order. Furthermore, if queues are sized properly, all tasks will meet the deadlines imposed by the systems, even if any of them incur a time penalty due to exceptional circumstances. As can be seen, the first three tasks are divided in four sub-tasks, one per range finder, that can also run in parallel. Thus, all stages can run in parallel, and in three of them, data gathered from the four range finders are processed concurrently.

The measurement pipeline of the proposed system is implemented in a single process using C\# and .NET Framework 4.0. Each element of the pipeline is implemented as a thread in the .NET thread pool: 14 threads implement the whole functionality of the measurement pipeline. Therefore, using a CPU with at least this number of cores ensures that all the elements of the pipeline can run in parallel. The last element of the pipeline, rail profile measurement, uses mathematical libraries programmed in $\mathrm{C}++$, due to their performance. These libraries are wrapped into the $\mathrm{C \#}$ project.

In this pipeline, the stages have unequal speeds. However, this is not a problem in the system since the most expensive stage is image acquisition (as will be shown in Section IV). Thus, given the fact that the first producer of the pipeline is the slowest one, the pipeline will never saturate.

\section{Laser line detection and extraction}

Laser line extraction is a straightforward task under controlled conditions of surrounding light and laser power [14]. However, common stripe extraction methods are highly sensitive to noise and they have major problems in industrial environments if they are not designed to deal with it correctly. In harsh environments a robust and accurate method capable of dealing with variable luminance, reflections which show up in images as noise, and uneven surfaces is required [15].

The PMS proposed in this paper extracts the singleline pattern projected onto the rail surface using a fourstep procedure based on a well-known differential geometric algorithm [16]. In the first step, the image is smoothed by means of a convolution with a Gaussian kernel to yield good results detecting salient lines in the image. In the second step, the derivatives of the Gaussian kernel are used to determine a quadratic polynomial for each point of the image, which is used to calculate the line direction for each pixel based on the second derivative. In the third step, once individual line points have been extracted, they are linked into lines. This task is carried out based on an hysteresis threshold: points with a second derivative smaller than $t_{l}$ are rejected; points with a second derivative larger than $t_{h}$ are accepted; and the rest of the points are accepted if they are connected to accepted points by a connected path. In the last step, if more than one line was detected in previous steps, they are linked since gaps in the line are present in the image. The linking procedure is based on the regression lines of the extracted lines, where $d$ is the maximum distance and $\alpha$ is the maximum angle allowed for lines to be linked. Therefore, five parameters must be fine tuned for the laser line extractor to properly detect the lines in the image: $\sigma, t_{l}, t_{h}, d$, and $\alpha$.

\section{E. Rail profile computation}

This is the first stage of the system where data provided by all range finders are synchronized (see Fig. 2), since the previous stages work as independent pipelines. In this stage 


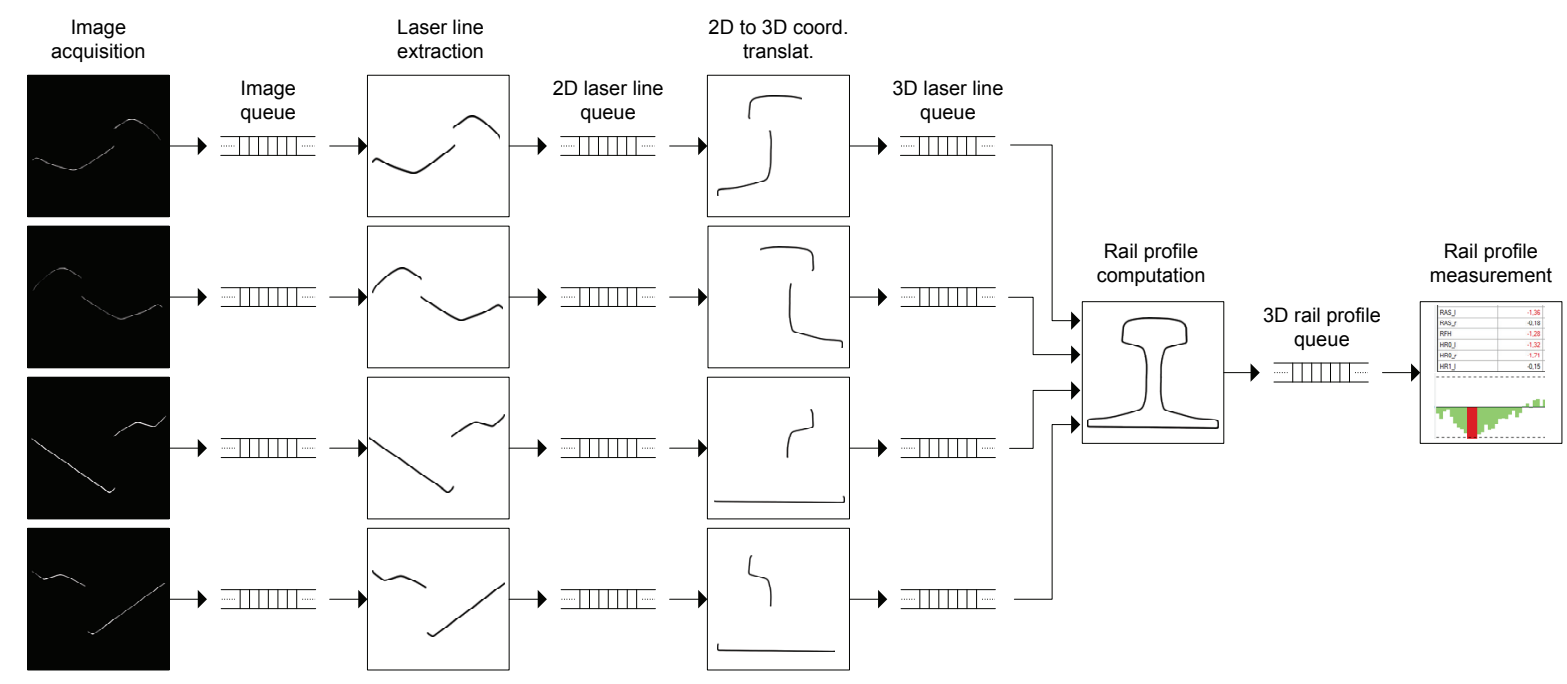

Fig. 2. Measurement pipeline of the proposed rail profile measurement system.

the partial rail contours generated by each range finder must be joined to create a rail profile at a specific $\mathrm{Z}$ coordinate of the rail track. Although the four pipelines connected to the four cameras of the system are identical, partial contours of the rail can reach this stage at slightly different speeds. Thus, a proper tagging and labelling method followed by a robust matching method are required to ensure that each partial contour is connected to other contours acquired at exactly the same $\mathrm{Z}$ coordinate of the rail track. Special attention must be paid if a frame is lost due to an external trigger failure, or if overflow in any queue of the pipeline is generated. These circumstances should never appear, but the proposed system is designed to automatically adapt to these issues, as described in [17].

Using the line extraction method described above, each range finder provides coordinates of the laser pattern projected onto the rail surface expressed in the coordinate system of its camera. In order to obtain metric measurements, points expressed in the coordinate system of the camera must be translated into the coordinate system of the world. Furthermore, since features extracted from several cameras are used to compute these measurements, a common reference system for all the range finders must be used. This reference system is computed in the second stage of the camera calibration procedure, i.e. in the calibration of extrinsic parameters, as will be described in Section III.

\section{F. Rail profile measurement}

The last stage of the pipeline provides metric measurements of the rail profile. It requires two steps. First, the rail data provided by the previous stage of the pipeline are aligned with a rail model. Second, the dimensions of the rail profile are computed. The output provided by the rail profile computation stage of the pipeline constitutes the data acquired from the rail profile expressed in the world coordinate system. This point cloud must be aligned with a rail model defined by the type of the rail-track that is being inspected. The alignment is performed by means of an efficient and robust registration method designed to accurately register $2 \mathrm{D}$ data to $\mathrm{CAD}$ models [18]. This method is an efficient variant of the ICP algorithm and aligns data obtained from standard structured light sensors

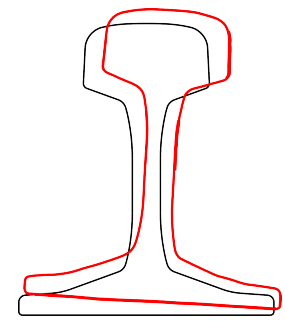

(a)

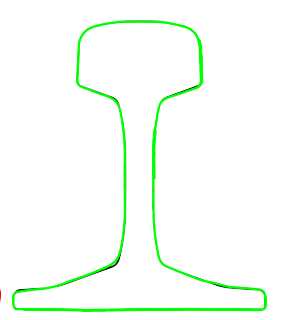

(b)
Fig. 3. Rail profile registration: (a) Point cloud and rail model; (b) Rail profile registered.

in less than one millisecond. It is based on geometric primitives on the 2D data rather than on points and uses a primitive caching method and an R-tree to accelerate the registration process. Fig. 3 shows the result of the registration process applied to the point cloud of a rail provided by the rail profile computation stage.

The rail primitives used in the registration process are listed in table I and are shown in Fig. 4 for both a symmetric and an asymmetric rail. These rail profile primitives are used to compute the rail profile dimensions listed in Table II. Fig. 5 shows several examples of rail profile dimensions computed using the rail profile primitives once the rail data has been registered to the rail model.

\section{CALIBRATION AND ASSESSMENT}

The profile measurement system proposed in this work must compute metric measurements of the rail under inspection to check its dimensional quality. Thus, the system must be calibrated to get correspondences of the image pixels in the camera coordinate system (CCS), with the 3D coordinate system of the scene, that is, the world coordinate system (WCS).

\section{A. Mathematical camera model}

Camera calibration is the procedure that determines the parameters that model the optical projection of a point in the 


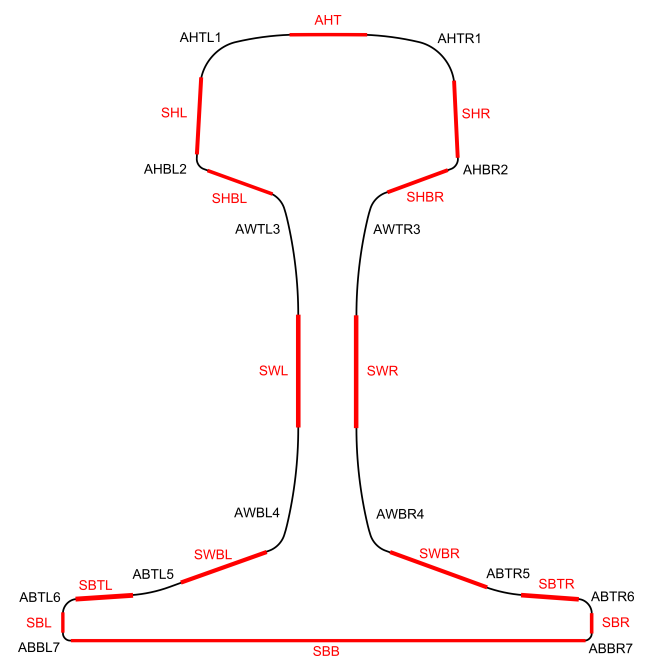

(a)

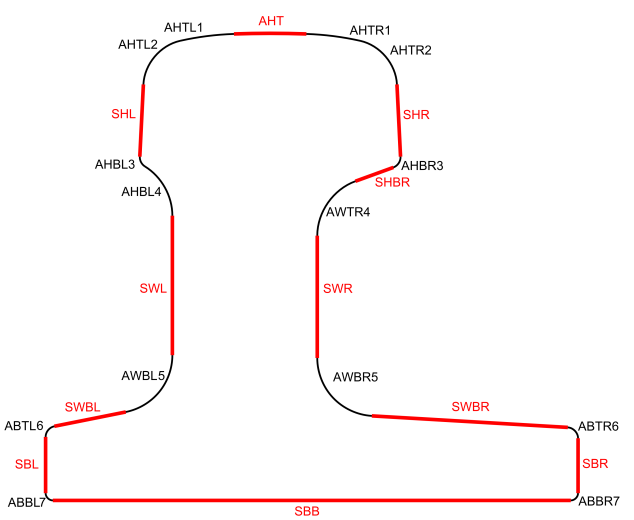

(b)

Fig. 4. Rail profile primitives: (a) Symmetric rail (rail model 60E1 in the standard UNE EN13674-1); (b) Asymmetric rail (rail model 54E1A1 in the standard UNE EN13674-1).

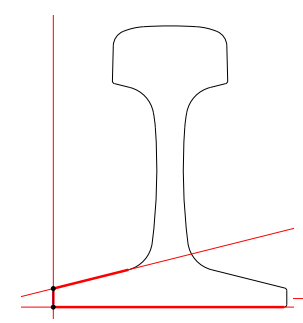

(a)

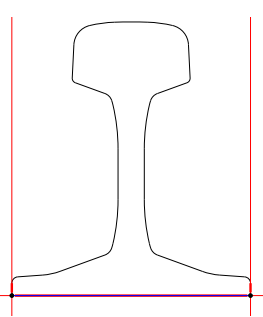

(b)

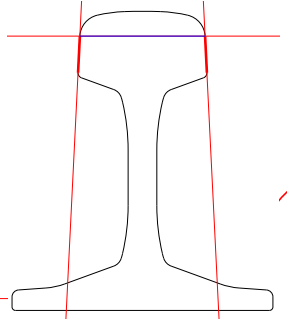

(c)

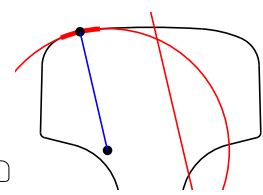

(d)

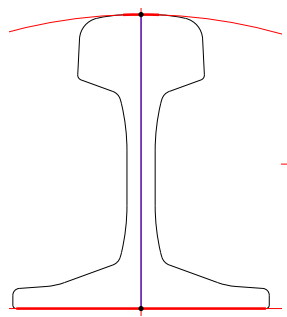

(e)

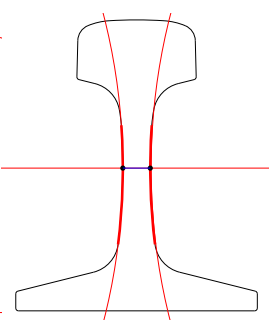

(f)

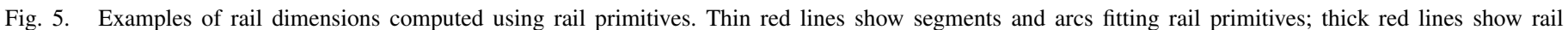

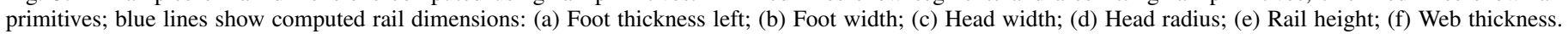

TABLE I. RAIL PROFILE PRIMITIVES

\begin{tabular}{ll}
\hline Primitive & Description \\
\hline AHT & Arc Head Top \\
AHTL, AHTR & Arc Head Top Left/Right \\
SHL, SHR & Segment Head Left/Right \\
AHBL, AHBR & Arc Head Bottom Left/Right \\
SHBL, SHBR & Segment Head Bottom Left/Right \\
AWTL, AWTR & Arc Web Left/Right \\
SWL, SWR & Segment Web Left/Right \\
AWBL, AWBR & Arc Web Bottom Left/Right \\
SWBL, SWBR & Segment Web Bottom Left/Right \\
ABTL, ABTR & Arc Base Top Left/Right \\
SBTL, SBTR & Segment Bottom Top Left/Right \\
ABTL, ABTR & Arc Bottom Top Left/Right \\
SBL, SBR & Segment Bottom Left/Right \\
ABBL, ABBR & Arc Bottom Bottom Left/Right \\
SBB & Segment Bottom Bottom \\
\hline
\end{tabular}

scene, $P\left(x^{w}, y^{w}, z^{w}\right)$, into a pixel (or a sub-pixel accuracy point) in the image, $P^{\prime}(r, c)$, as shown in Fig. 6 . The internal characteristics of the camera, such as sensor pixel size and lens distortions, are described by means of intrinsic parameters, whereas position and orientation of the camera are described by means of extrinsic parameters [19].

The calibration of the range finders that constitute the proposed PMS follows the non-linear camera model proposed
TABLE II. RAIL PROFILE DIMENSIONS

\begin{tabular}{ll}
\hline Dimension & Description \\
\hline RH & Rail Height \\
RFH & Rail Flange Height \\
FW & Foot Width \\
HF & Head Form \\
HW & Head Width \\
HWA & Head Width (Arema standard) \\
FT_1, FT_r & Foot Thickness left/right \\
RAS_1, RAS_r & Rail Asymmetry left/right \\
WT & Web Thickness \\
HR & Head Radius \\
FC & Foot Concavity \\
\hline
\end{tabular}

in [20], that maps 3D world coordinates $\left(x_{j}^{w}, y_{j}^{w}, z_{j}^{w}\right)$ to pixel coordinates in the 2D camera sensor $\left(r_{j}, c_{j}\right)$, where $j$ identifies the range finder $(j \in[1,4])$, by means of several transformations. The calibration procedure following this model is described in depth for a single laser range finder in [21].

Calibrating a 3D imaging system based on active triangulation using an area sensor involves computing the following three sets of parameters. The first comprises the intrinsic parameters of the camera model: $\mathcal{I}=\left\{f, s^{x}, s^{y}, u_{0}, v_{0}, k_{1}, k_{2}, k_{3}, p_{1}, p_{2}\right\}$, where $f$ is the effective focal length; $s^{x}$ and $s^{y}$ are the image scale factors; 


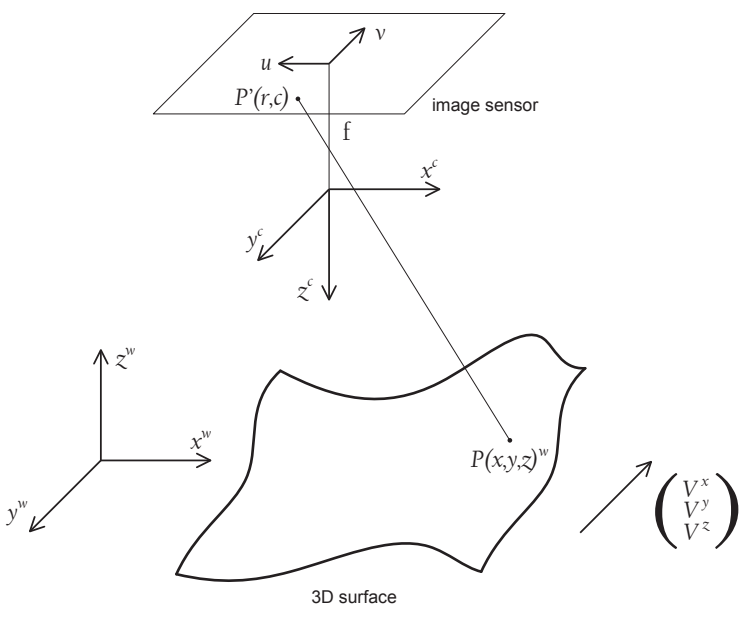

Fig. 6. Camera and perspective projection.

$u_{0}$ and $v_{0}$ represent the principal point of the camera, also called the image center; $k_{1}, k_{2}$ and $k_{3}$ are the coefficients for radial distortions; and $p_{1}$ and $p_{2}$ are coefficients for tangential distortions. The second comprises the extrinsic parameters of the camera model: $\mathcal{E}=\left\{\alpha, \beta, \gamma, t_{1}, t_{2}, t_{3}\right\}$, where $\alpha, \beta$ and $\gamma$ are the Euler angles in the rotation matrix; and $t_{1}, t_{2}$ and $t_{3}$ are the coefficients of the translation vector. The third set comprises the components of the motion vector of the object under inspection: $\mathcal{V}=\left\{V^{x}, V^{y}, V^{z}\right\}$.

The camera calibration procedure requires an array of precisely known 3D target points, or reference points, $\left\{W=\left(X_{i}^{W}, Y_{i}^{W}, Z_{i}^{W}\right), i \in[1, n]\right\}$, acquired from a calibrated pattern located in the scene. The 16 parameters of the mathematical model of each camera, $\mathcal{M}_{j}=\left\{\mathcal{I}_{j} \cup \mathcal{E}_{j}\right\}, j \in[1,4]$, are estimated based on the array with corresponding feature points in the image sensor of the $j$-th camera, $C_{j}=\left\{\left(X_{i}^{C_{j}}, Y_{i}^{C_{j}}\right), i \in[1, n], j \in[1,4]\right\}$. The estimation task relies on solving the non-linear least-squares problem shown in Eq.(1) for each range finder.

$$
\min _{\mathcal{M}_{j} \in \mathbb{R}^{16}} \mathcal{F}_{j}\left(\mathcal{M}_{j}, C_{j}\right)=\sum_{i=1}^{n}\left(\left(x_{i, j}^{c}-r_{i, j}\right)^{2}+\left(y_{i, j}^{c}-c_{i, j}\right)^{2}\right)
$$

The calibration process of the proposed PMS involves calibrating four laser range finders. This process is similar to the calibration of a single range finder, but the calibration pattern must be in the field of view of all four cameras.

Once the mathematical camera model is solved, the transformation from $3 \mathrm{D}$ world coordinates to $2 \mathrm{D}$ image sensor coordinates can be carried out. The transformation from image coordinates to world coordinates is a simple procedure: the light plane, that is, the plane on which the single-line pattern fluctuates due to the shape of the product surface, is computed based on several images acquired with the calibrated pattern in the scene, one from each camera. Then the system is able to provide the $3 \mathrm{D}$ coordinates of a point in the scene based on a coordinate system fixed to this plane.

The length of the manufactured rail can be measured once the rail movement in the manufacturing line is calibrated. Since

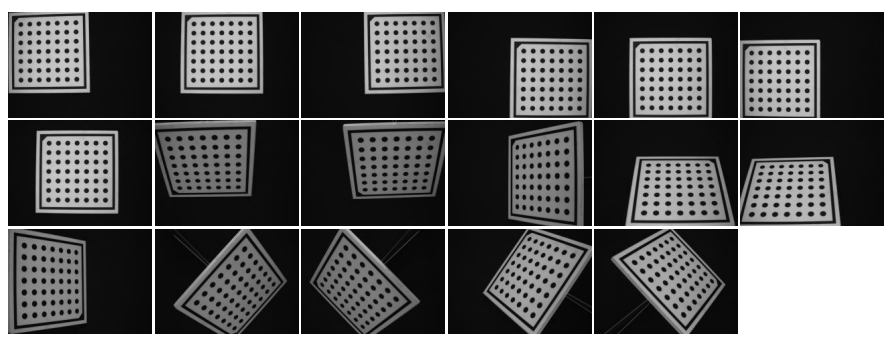

Fig. 7. Images for intrinsic parameter calibration.

the rail mill forces the rail to move along the longitudinal axis of the line, $V^{x}$ and $V^{y}$ will tend to be null. On the other hand, $V^{z}$ will depend on the speed of the rail movement while manufacturing. Therefore, calibrating the speed of the rail movement requires calibrating the rotary encoder that supervises this movement.

\section{B. Calibration setup}

The internal parameters and distortions of the camera are estimated by means of a planar-based calibration. A checkerboard pattern is imaged in several orientations and distances from the camera to identify a unique solution for $\mathcal{I}=\left\{f, s^{x}, s^{y}, u_{0}, v_{0}, k_{1}, k_{2}, k_{3}, p_{1}, p_{2}\right\}$. Fig. 7 shows the set of images acquired to estimate the internal parameters of a camera of the proposed PMS. The reference points are the centers of the circles printed in the pattern. The manufacturing procedure of the calibration plate guarantees that the locations of reference points have an error of less than $50 \mu \mathrm{m}$, which gives an uncertainty of $29 \mu \mathrm{m}$, computed as type B uncertainty evaluation [22].

The set of extrinsic parameters for each range finder is computed using a calibration pattern consisting of a flat aluminium surface with 13 protruding cylinders. The reference points are the center of each cylinder and its radius. Fig. 8a shows the calibration pattern illuminated by the laser emitters of the system in a laboratory setup. An example of images acquired by the four cameras of the system when the calibration pattern is illuminated by the laser line emitters can be seen in Fig. 8b This calibration pattern is also used in the rail mill for computing the extrinsic parameters of the camera models. The manufacturing procedure of the calibration object guarantees that the locations of the cylinders have an error of less than $50 \mu \mathrm{m}$, which gives an uncertainty of $29 \mu \mathrm{m}$, computed as type B uncertainty evaluation [22].

Laser lines are detected and extracted in the same way as described for the case of rail profile extraction (see section II-D). Fig. 8c shows the laser lines extracted from images in Fig. 8b. Said lines are then fitted to ellipses by the direct least square method. Ellipses are then filtered according to their likelihood to correspond to actual calibration cylinders, taking into account for each ellipse both its radius ratio and the length of the laser line in relationship with its shape. Ellipses that are rejected by either of these two filters are discarded. Then, an attempt is made to associate each of the ellipses that passed the filters with one of the calibration cylinders. This is done in four steps. In the first step, the centermost ellipse is found and associated with the centermost cylinder. In the second step, the coordinates of the ellipse centers are rotated by a predefined 


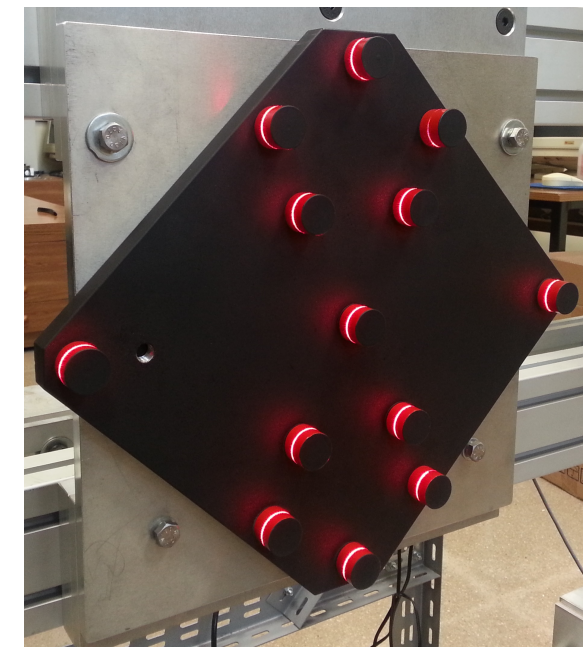

(a)
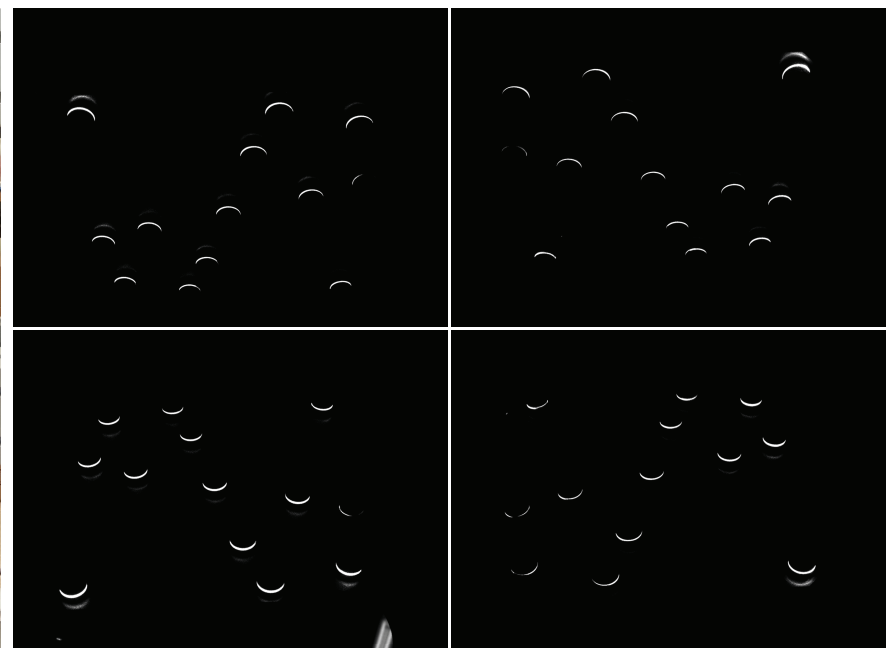

(b)
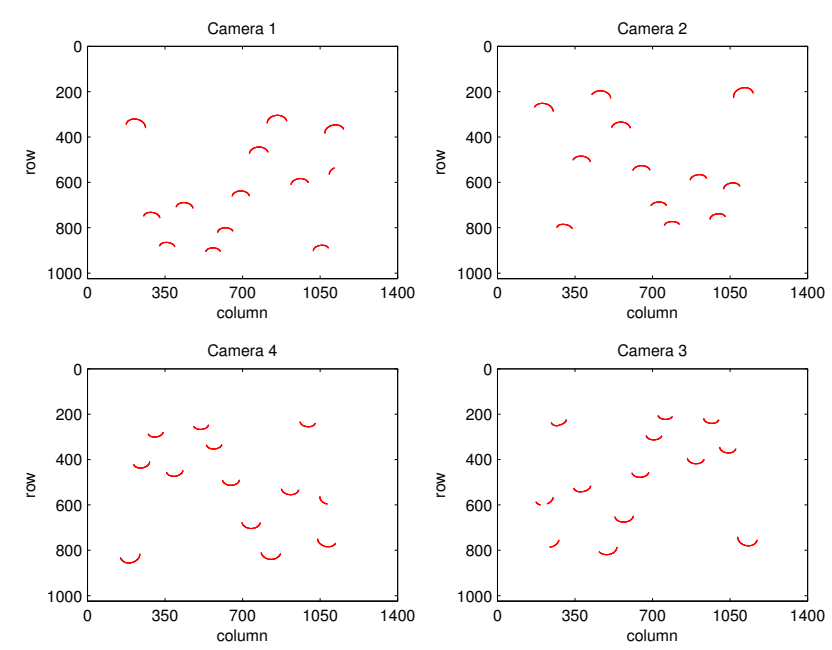

(c)

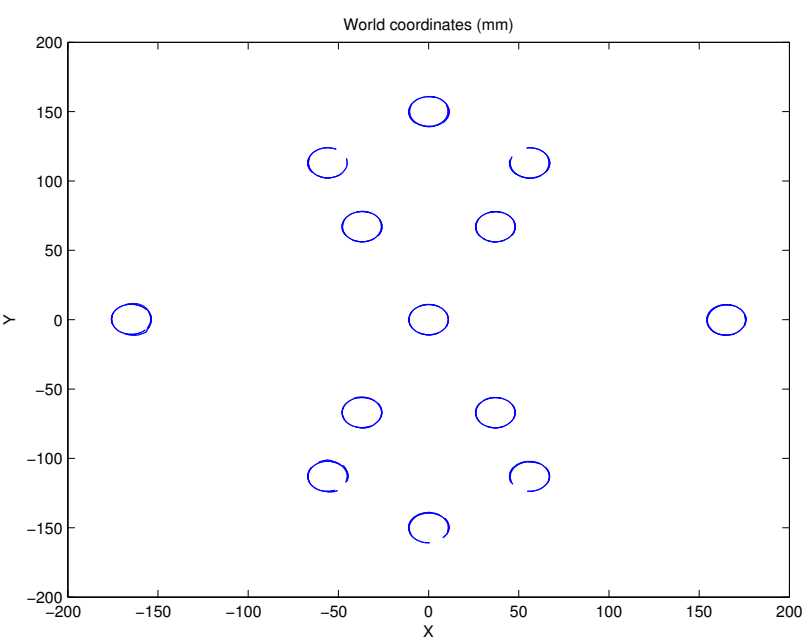

(d)

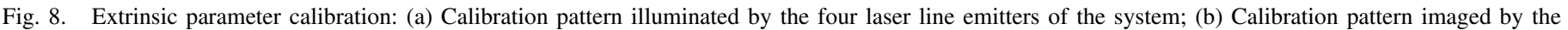

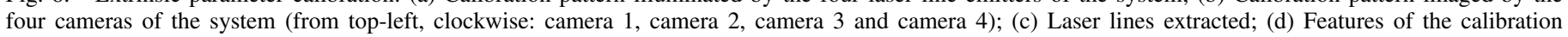
pattern expressed in world coordinates.

amount in order to roughly compensate for the camera roll angle. In the third step, ellipse coordinates are constrained to the range $[-1,1]$ in such a way that the centermost point is assigned the coordinates $(0,0)$, negative $X$ coordinates are divided by the absolute value of the lowest (furthest from the center) negative $X$ coordinate, positive $X$ coordinates are divided by the value of the highest positive $X$ coordinate and so on. Actual cylinder coordinates are constrained in the same way. In the fourth step, the coordinates of the ellipses are compared to those of the cylinders, and each ellipse is associated with the cylinder which is closest in Euclidean distance, unless another ellipse is closer to the same cylinder, in which case the first one is discarded. Ellipses whose distance to the cylinders is greater than an imposed threshold, $t_{e c}$, are also discarded. Correspondences between the (original, unconstrained) center coordinates of the remaining ellipses and the coordinates of their associated cylinders are then used to compute a pose. Fig. 8d shows the laser lines extracted by the four range finders and expressed in the WCS using the calibration data computed as described above.

Before acquiring images with the calibration pattern in the scene the system must be set up to properly extract the single-line pattern from the acquired images. Thus, system calibration depends on parameter selection in the set-up process. In order to choose the best suited set of parameters, a factorial experiment was carried out. Different values for the acquisition time, the standard deviation of the Gaussian for the smoothing kernel, the distance limit for cylinder matching and the maximum number of ellipses to use in the pose computation, along with multiple variations of the direct least square method for ellipse fitting, were considered. Based on this experiment, two main parameters are identified as the main influence in the laser line extraction from the calibration pattern: the acquisition time, $t$, and the standard deviation of the Gaussian for the smoothing kernel, $\sigma$. 


\section{Calibration assessment}

In order to evaluate the accuracy of the camera calibration procedure described above, reprojection errors and the estimated focal length are shown to assess the calibration of internal parameters, and three different metrics computed over the calibration template are provided to assess the calibration of external parameters.

In the process used to obtain the internal parameters, the radial lens distortions was modelled using two models: analytical and numerical. The former uses only one parameter to model radial distortions, $\kappa$, whereas the latter is more complex and uses three parameters, $k_{1}, k_{2}, k_{3}$, as described in Section III-A. Although the accuracy achieved with the former is lower, it can be inverted analytically whereas the latter can only be solved numerically. Table III shows the reprojection errors for each camera of the system computed using these two models. The reprojection error expresses the average distance, in pixels, between the backprojected reference points of the calibration pattern and their extracted coordinates in the image plane. As can be seen, the largest reprojection error computed using a numerical model it is 0.16 pixels, whereas using an analytical model is below 0.17 pixels. In both cases, this ensures that the calibration process was successful. Although there is only a slight difference between the results of the two models, the calibration parameters obtained by the numerical will be used in the system. In addition, the estimated focal length of the lens of each camera is computed and shown in the table (the nominal focal length of each lens is $16 \mathrm{~mm}$; see section II-B).

TABLE III. CALIBRATION ASSESSMENT: INTERNAL PARAMETERS

\begin{tabular}{lccc}
\hline Camera & $\begin{array}{c}\text { Distortion } \\
\text { model }\end{array}$ & $\begin{array}{c}\text { Reprojection error } \\
\text { (pixels) }\end{array}$ & $\begin{array}{c}\text { Focal length } \\
(\mathrm{mm})\end{array}$ \\
\hline \multirow{2}{*}{ Camera \#1 } & Analytical & 0.154 & 16.268 \\
& Numerical & 0.141 & 16.269 \\
Camera \#2 & Analytical & 0.164 & 16.225 \\
& Numerical & 0.154 & 16.231 \\
Camera \#3 & Analytical & 0.166 & 16.082 \\
& Numerical & 0.160 & 16.082 \\
Camera \#4 & Analytical & 0.169 & 16.143 \\
& Numerical & 0.163 & 16.144 \\
\hline
\end{tabular}

Three different metrics were designed to assess the computation of external parameters: center distance (CD), radius difference (RD), and point distance (PD). The CD metric expresses the distance between the center of each cylinder in the calibration template and the center of each cylinder computed based on the image acquired by each laser range finder. The RD metric expresses the difference between the radius of each cylinder in the calibration template and the radius computed by the system based on images acquired by each range finder. The PD metric expresses the difference between the radius of each cylinder and the distance from the computed center of the cylinder to each of the sampled points of the cylinder. Table IV shows the errors of the external calibration of each range finder.

\section{EXPERIMENTAL RESULTS AND DISCUSSION}

The accuracy evaluation of rail profile measurements provided by the proposed PMS was examined using a prototype first in the laboratory and then in a rail rolling mill courtesy of ArcelorMittal Asturias. A randomly selected rail was inspected
TABLE IV. CALIBRATION ASSESSMENT: EXTERNAL PARAMETERS (UNITS IN MM)

\begin{tabular}{lccccccccc}
\hline \multirow{2}{*}{ Camera } & \multicolumn{3}{c}{ CD } & & \multicolumn{3}{c}{ RD } & \multicolumn{3}{c}{ PD } \\
& mean & STD & max. & mean & STD & max. & mean & STD & max \\
\hline Camera \#1 & 0.176 & 0.035 & 0.221 & 0.101 & 0.038 & 0.158 & 0.097 & 0.015 & 0.116 \\
Camera \#2 & 0.159 & 0.058 & 0.271 & 0.076 & 0.053 & 0.194 & 0.123 & 0.034 & 0.180 \\
Camera \#3 & 0.299 & 0.031 & 0.343 & 0.047 & 0.019 & 0.065 & 0.226 & 0.040 & 0.297 \\
Camera \#4 & 0.185 & 0.069 & 0.274 & 0.169 & 0.049 & 0.250 & 0.106 & 0.035 & 0.175 \\
\hline
\end{tabular}

TABLE V. ACCURACY OF DIMENSIONAL INSPECTION (UNITS IN MM)

\begin{tabular}{|c|c|c|c|c|c|c|c|}
\hline \multirow{2}{*}{ Dim. } & \multirow{2}{*}{ Length } & \multicolumn{5}{|c|}{ PMS-GT } & \multirow{2}{*}{ MP-GT } \\
\hline & & $\# 1$ & $\# 2$ & $\# 3$ & $\# 4$ & $\# 5$ & \\
\hline HW & 10000 & -0.02 & -0.04 & -0.06 & -0.04 & -0.04 & 0.15 \\
\hline FW & 10000 & 0.01 & -0.01 & -0.03 & -0.02 & -0.03 & - \\
\hline $\mathrm{RH}$ & 10000 & -0.08 & -0.10 & -0.09 & -0.11 & -0.11 & - \\
\hline HW & 20000 & 0.05 & 0.04 & 0.04 & 0.04 & 0.03 & 0.15 \\
\hline FW & 20000 & 0.03 & 0.02 & 0.03 & 0.03 & 0.03 & - \\
\hline RH & 20000 & -0.16 & -0.16 & -0.12 & -0.12 & -0.12 & - \\
\hline HW & 30000 & 0.03 & 0.03 & 0.03 & 0.04 & 0.01 & 0.07 \\
\hline FW & 30000 & -0.05 & -0.04 & -0.06 & -0.05 & -0.05 & - \\
\hline $\mathrm{RH}$ & 30000 & -0.15 & -0.19 & -0.14 & -0.14 & -0.17 & - \\
\hline HW & 40000 & 0.01 & 0.01 & 0.01 & 0.00 & 0.01 & 0.05 \\
\hline FW & 40000 & 0.00 & 0.04 & 0.00 & 0.02 & 0.01 & - \\
\hline RH & 40000 & -0.19 & -0.19 & -0.20 & -0.19 & -0.19 & - \\
\hline HW & 50000 & -0.01 & 0.00 & 0.00 & 0.01 & 0.00 & 0.08 \\
\hline FW & 50000 & 0.03 & 0.03 & 0.02 & -0.01 & 0.00 & - \\
\hline RH & 50000 & -0.19 & -0.19 & -0.20 & -0.20 & -0.19 & - \\
\hline HW & 60000 & 0.00 & 0.00 & 0.00 & -0.01 & 0.00 & 0.13 \\
\hline FW & 60000 & 0.01 & -0.01 & -0.02 & -0.01 & -0.03 & - \\
\hline RH & 60000 & -0.13 & -0.12 & -0.11 & -0.12 & -0.07 & - \\
\hline
\end{tabular}

five consecutive times under the same working conditions. The model of the rail is $60 \mathrm{E} 1$, according to the standard UNE EN13674-1. This rail track was also inspected in the rail rolling mill with a Greenwood MiniProf Rail gauge ${ }^{1}$ featuring an accuracy better than $\pm 11 \mu \mathrm{m}$ and a repeatability of $\pm 2.5 \mu \mathrm{m}$. Once the rail was inspected in the mill, it was cut into pieces, and several specimens were measured in the metrology lab of the R\&D Centre of ArcelorMittal in Asturias using a Trimos Temp 1282 coordinate-measuring machine (CMM) featuring an accuracy better than $\pm 1 \mu \mathrm{m}$ in FW and better than $\pm 20 \mu \mathrm{m}$ in HW and RH. Dimensions provided by the MiniProf gauge and the CMM are used to assess the dimensions computed by the PMS.

Table V shows differences among the measurements computed by the dimensional inspection system proposed in this paper, PMS (five repetitions of the experiment), and the measurements carried out by the technicians in the metrology lab, considered the ground truth, GT. Also, differences with the dimension provided by the MiniProf Rail gauge, MP, are shown. As can be seen, errors provided by the system are between $-0.05 \mathrm{~mm}$ and $0.20 \mathrm{~mm}$. This means that dimensions provided by the proposed PMS for HW, FW and RH have an error of less than $0.12 \%$

In addition to accuracy, in order to evaluate the performance of the PMS, a repeatability evaluation is carried out to assess the consistency of the system. The repeatability evaluation expresses the range of measurements provided for the same dimension of the same rail specimen under consistent conditions. This test was carried out using the same method, on

\footnotetext{
${ }^{1}$ https://www.greenwood.dk/miniprofrail.php
} 


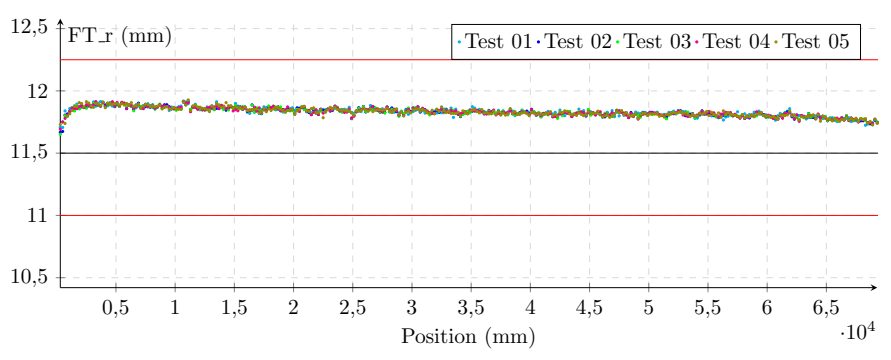

(a)

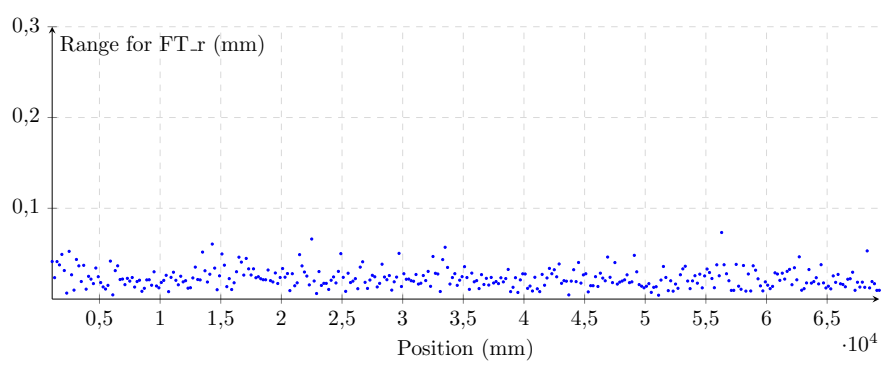

(b)

Fig. 9. Results of the repeatability evaluation for Foot Thickness right (FT_r): (a) Computed values (the black horizontal line is the expected value, while the red horizontal lines are the maximum and minimum tolerated values); (b) Measurement range.

TABLE VI. REPEATABILITY OF DIMENSIONAL INSPECTION (UNITS IN MM)

\begin{tabular}{ccccccccccc}
\hline Length & FC & FT_1 & FT_r & FW & HW & HWA & RAS_1 & RAS_r & RH & WT \\
\hline 10000 & 0.018 & 0.041 & 0.024 & 0.039 & 0.029 & 0.039 & 0.109 & 0.108 & 0.030 & 0.060 \\
20000 & 0.013 & 0.046 & 0.026 & 0.035 & 0.021 & 0.016 & 0.099 & 0.108 & 0.038 & 0.071 \\
30000 & 0.010 & 0.039 & 0.024 & 0.029 & 0.018 & 0.015 & 0.085 & 0.100 & 0.041 & 0.055 \\
40000 & 0.012 & 0.037 & 0.023 & 0.032 & 0.018 & 0.014 & 0.098 & 0.105 & 0.025 & 0.048 \\
50000 & 0.012 & 0.043 & 0.022 & 0.036 & 0.020 & 0.014 & 0.095 & 0.108 & 0.015 & 0.065 \\
60000 & 0.016 & 0.049 & 0.022 & 0.031 & 0.021 & 0.017 & 0.084 & 0.096 & 0.032 & 0.049 \\
\hline
\end{tabular}

the same item, in the same installation, by the same operator and using the same system within short intervals of time, according to ISO 5725-1:1994. Fig. 9 shows an example of the repeatability evaluation. Fig. 9(a) shows the computed values for the five consecutive inspections of the rail for FT_r, and Fig. 9(b) shows the range of the measurements for this dimension in the five runs. Red lines indicate the lower and upper limit imposed by the specification to which the rail is manufactured; the black line is the expected value according to the specification. Table VI shows the range of all the dimensions computed by the system, expressed as the average of the ranges each $10 \mathrm{~m}$ of the rail. As can be seen, the system is highly repeatable.

Finally, the running time of the system is analysed. The host computer used by the PMS is an HP Z620 Workstation equipped with an Intel Xeon E5-2643 v2 processor (6 cores @ $3.5 \mathrm{GHz}$ ) and $12 \mathrm{~GB}$ of RAM memory. The elapsed time in each stage of the pipeline was measured in ten consecutive rails inspected in the rail mill. Table VII shows the computing times: mean, standard deviation (SD) and the upper limit of the 99\% confidence interval (UCL). Taking this information into account, the system is able to work with an acquisition rate up to $210 \mathrm{fps}$. In the aforementioned experiments the acquisition time of the cameras was set to $15 \mathrm{~ms}$ after several experiments with different surface conditions of rails and lens apertures.
Thus, the acquisition rate was limited to $66 \mathrm{fps}$.

TABLE VII. RUNNING TIMES (UNITS IN MS)

\begin{tabular}{lcccc}
\hline & $\begin{array}{c}\text { Laser line } \\
\text { extraction }\end{array}$ & $\begin{array}{c}\text { 2D to 3D } \\
\text { translation }\end{array}$ & $\begin{array}{c}\text { Rail profile } \\
\text { computation }\end{array}$ & $\begin{array}{c}\text { Rail profile } \\
\text { measurement }\end{array}$ \\
\hline mean & 4.62 & 0.01 & 0.02 & 3.09 \\
SD & 0.44 & $<0.01$ & $<0.01$ & 1.72 \\
$99 \%$ UCL & 4.68 & 0.01 & 0.03 & 3.16 \\
\hline
\end{tabular}

\section{CONCLUSIONS}

In modern rail rolling mills, accurate and repeatable profile measurements are crucial for inspecting and assessing rails that will be used in the transport sector by high-speed trains and in industry by heavy-loaded trains. The system proposed in this paper uses four conventional, inexpensive laser range finders to measure transverse sections of rails. The range finders are calibrated following a non-linear camera model using a common reference to properly express the point clouds in the world coordinate system. Then point clouds are aligned to the rail model by means of an efficient and robust registration method.

The experiments carried out in the laboratory and in a rail rolling mill demonstrate the accuracy and repeatability of the system. Depending on the dimension, errors provided by the system are between $-0.05 \mathrm{~mm}$ and $0.20 \mathrm{~mm}$, with a maximum error of less than $0.12 \%$. Furthermore, all parallelizable tasks were designed and developed to be executed concurrently. Thus, the proposed rail profile measurement system is able to work with an acquisition rate of up to $210 \mathrm{fps}$, that allows capturing high density 3D data of the rail.

\section{ACKNOWLEDGMENT}

This work was partially funded by the projects TIN201124903 and TIN2014-56047-P of the Spanish National Plan for Research, Development and Innovation, and by the project FUO-EM-269-13.

The authors would like to thank the technicians of ArcelorMittal Asturias for their helpful assistance during tests.

\section{REFERENCES}

[1] E. Malamas, E. Petrakis, M. Zervakis, L. Petit, and J. Legat, "A survey on industrial vision systems, applications and tools," Image and Vision Computing, vol. 21, no. 2, pp. 171-188, 2003.

[2] F. Blais, "Review of 20 years of range sensor development," Journal of Electronic Imaging, vol. 13, no. 1, pp. 231-243, 2004.

[3] G. Sansoni, M. Trebeschi, and F. Docchio, "State-of-the-art and applications of 3D imaging sensors in industry, cultural heritage, medicine, and criminal investigation," Sensors, vol. 9, pp. 568-601, 2009.

[4] R. Ahlers and J. Lu, "Stereoscopic vision: an application-oriented overview," in SPIE Optics, Illumination, and Image Sensing for Machine Vision, 1989, pp. 298-308.

[5] M. Papaelias, C. Roberts, and C. Davis, "A review on non-destructive evaluation of rails: State-of-the-art and future development," in Proceedings of the Institution of Mechanical Engineers Part F Journal of Rail and Rapid Transit, vol. 222, no. 4, 2008, pp. 367-384.

[6] Q. Tang, "Railway track geometry realtime inspection system," in Proceedings of the IEEE Instrumentation and Measurement Technology Conference, 1992, pp. 656-660. 
[7] C. Alippi, E. Casagrande, F. Scotti, and V. Piuri, "Composite realtime image processing for railways track profile measurement," IEEE Transactions on Instrumentation and Measurement, vol. 49, no. 3, pp. 559-564, 2000.

[8] F. Attivissimo, A. Danese, N. Giaquinto, and P. Sforza, "A railway measurement system to evaluate the wheel-rail interaction quality," IEEE Transactions on Instrumentation and Measurement, vol. 56, no. 5, pp. 1583-1589, 2007.

[9] S. Magnus and D. Magnus, "Rail measurement system," Mar. 19 2009, US Patent App. 12/229,244.

[10] Z. Liu, J. Sun, H. Wang, and G. Zhang, "Simple and fast rail wear measurement method based on structured light," Optics and Lasers in Engineering, vol. 49, no. 11, pp. 1343-1351, 2011.

[11] K. Xu, P. Zhou, and C. Hu, "3d detection technique of surface defects for heavy rail based on binocular stereo vision," vol. 8417, 2012, pp. 841 707-841 707-5.

[12] J. Molleda, R. Usamentiaga, A. Millara, D. Garcia, P. Manso, C. Suarez, and I. Garcia, "A profile measurement system for rail manufacturing using multiple laser range finders," in 2015 IEEE Industry Applications Society Conference, vol. 1. IEEE, 2015, pp. 1-8.

[13] J. Molleda, R. Usamentiaga, and D. Garcia, "On-line flatness measurement in the steelmaking industry," Sensors, vol. 13, no. 8, pp. 10245$10272,2013$.

[14] M. Levoy, S. Rusinkiewicz, M. Ginzton, J. Ginsberg, K. Pulli, D. Koller, S. Anderson, J. Shade, B. Curless, L. Pereira, J. Davis, and D. Fulk, "The digital michelangelo project: 3d scanning of large statues," in Proceedings of the ACM SIGGRAPH Conference on Computer Graphics. ACM Press/Addison-Wesley Publishing Co. New York, NY, USA, 2000, pp. 131-144.

[15] R. Usamentiaga, J. Molleda, and D. Garcia, "Fast and robust laser stripe extraction for 3d reconstruction in industrial environments," Machine Vision and Applications, vol. 23, pp. 179-196, 2012, jCR: 1.103 [2012]. [Online]. Available: http://dx.doi.org/10.1007/s00138-010-0288-6

[16] C. Steger, "An unbiased detector of curvilinear structures," IEEE Transactions on Pattern Analysis and Machine Intelligence, vol. 20, no. 2, pp. 113-125, 1998.

[17] J. Molleda, R. Usamentiaga, D. Garcia, and F. Bulnes, "Towards autonomic computing in machine vision applications: techniques and strategies for in-line $3 \mathrm{~d}$ reconstruction in harsh industrial environments," in Proceedings of 2011 SPIE Electronic imaging. Image Processing: Machine Vision Applications, vol. 7877, 2011, pp. 1-13.

[18] R. Usamentiaga, D. Garcia, and J. Molleda, "Efficient registration of $2 \mathrm{D}$ points to CAD models for real-time applications," Journal of Real-Time Image Processing, pp. 1-19, 2015, jCR: 1.111 - T2 [2013]. [Online]. Available: http://dx.doi.org/10.1007/s11554-015-0485-7

[19] R. Tsai, "A versatile camera calibration technique for high accuracy $3 \mathrm{~d}$ machine vision metrology using off-the-shelf tv cameras and lenses," IEEE Journal of Robotics and Automation, vol. 3, no. 4, pp. 323-344, 1987.

[20] J. Heikkilä and O. Silven, "A four-step camera calibration procedure with implicit image correction," in Proceedings of the IEEE Computer Society Conference on Computer Vision and Pattern Recognition, 1997, pp. 1106-1112.

[21] J. Molleda, R. Usamentiaga, D. Garcia, and F. Bulnes, "Real-time flatness inspection of rolled products based on optical laser triangulation and three-dimensional surface reconstruction," Journal of Electronic Imaging, vol. 19, no. 3, pp. 1-14, 2010, article id. 031206.

[22] Evaluation of measurement data - Guide to the expression of uncertainty in measurement, JCGM, 2008. 